\title{
Avaliação de Serviços de Saúde Mental Brasileiros: satisfação dos usuários e fatores associados
}

\author{
Brazilian Mental Health Services Assessment: \\ user satisfaction and associated factors
}

Sarah Nascimento Silva ${ }^{1}$ Marina Guimarães Lima ${ }^{2}$ Cristina Mariano Ruas ${ }^{2}$
${ }^{1}$ Programa de PósGraduação em Medicamentos e Assistência Farmacêutica, Departamento de Farmácia Social, Faculdade de Farmácia, Universidade Federal de Minas Gerais (UFMG). Av. Presidente Antônio Carlos 6627, Pampulha. 31270-901 Belo Horizonte MG Brasil. sarahnsilva@gmail.com ${ }^{2}$ Departamento de Farmácia Social, UFMG. Belo

Horizonte MG Brasil.
Abstract Patient satisfaction is an important criterion for assessing the quality of Brazilian mental health services at Psychosocial Care Centers (CAPS - from the Portuguese 'Centro de Atenção Psicossocial'). The aim of this study was to evaluate the satisfaction of users at the main CAPS in a region of Minas Gerais state, Brazil, as well as associated factors. This was a cross-sectional study with 11 CAPS. Patients were interviewed using the Patient Satisfaction with Mental Health Services Scale (SATIS-BR) and a semi-structured questionnaire containing sociodemographic and clinical variables. The users were satisfied with the CAPS, particularly in terms of staff competence and the welcome received and care provided. The physical facilities and comfort at the centers obtained the lowest satisfaction scores on the scale. Almost half of the users were unfamiliar with basic aspects of their drug therapy, such as the name of medicines, and one-third reported inappropriate use of medications. Users of midsize CAPS and those providing treatment for alcohol and drug addiction were more satisfied than patients at mental health or 24-hour CAPS. Although this study identified a need for improvement in physical facilities, mechanisms of participation and patient empowerment, the users were satisfied with the CAPS care model.

Key words Health service evaluation, Mental health services, Patient satisfaction
Resumo A satisfação dos usuários é um importante critério para avaliar a qualidade dos Centros de Atenção Psicossocial (CAPS). O objetivo foi avaliar a satisfação de usuários dos principais CAPS de uma região de Minas Gerais e seus fatores associados. Foi realizado um estudo transversal em 11 CAPS, os usuários foram entrevistados para aplicação da Escala de Avaliação da Satisfação dos Usuários com os Serviços de Saúde Mental e um formulário semi-estruturado com variáveis sociodemográficas e clinicas. Os usuários estavam satisfeitos com os CAPS, sobretudo quanto à competência dos profissionais, acolhida e ajuda recebida no serviço. Condições físicas e conforto do serviço obtiveram os menores escores na escala de avaliação de satisfação. Verificou-se que quase metade dos usuários não conhecia aspectos básicos de sua terapia medicamentosa, como o nome dos medicamentos em uso, e um terço relatou que já fez uso inadequado destes. Os usuários dos CAPS álcool e drogas ou de serviços de médio porte estavam mais satisfeitos que os dos CAPS saúde mental ou serviços com funcionamento $24 \mathrm{~h}$. Os usuários estão satisfeitos com o modelo de atenção praticado nos CAPS, embora detectada a necessidade de melhorias na estrutura física, mecanismos de participação e empoderamento dos usuários.

Palavras-chave Avaliação de serviços de saúde, Serviços de saúde mental, Satisfação do paciente 


\section{Introdução}

Os Centros de Atenção Psicossocial (CAPS) constituíram a principal estratégia da política de saúde mental para substituir o modelo asilar e hospitalocêntrico, redefinindo os conceitos sobre o tratamento da saúde mental. Essa política, ao propor o tratamento em liberdade, promove a inclusão dos usuários como sujeitos ativos em seu tratamento, em uma proposta multidisciplinar que considera a história, a cultura e o cotidiano na construção de um Projeto Terapêutico Singular (PTS $)^{1}$. Nesse sentido, a avaliação da satisfação dos usuários com o serviço é um importante critério para avaliação da qualidade dos CAPS, na medida em que considera a perspectiva do usuário.

Os CAPS desempenham o papel de porta de entrada no Sistema Único de Saúde (SUS), estão organizados nas modalidades CAPS I, II e III, de acordo com o porte, a complexidade e a abrangência populacional. Modalidades específicas complementam atendimento às crianças e adolescentes (CAPSi), e os CAPS oferecem tratamento a adultos, crianças ou adolescentes com transtornos decorrentes do uso e dependência de álcool e outras drogas ${ }^{2}$. A criação de instrumentos legais foi fundamental para consolidação e desenvolvimento da Política de Saúde Mental criada em 2001. Em 2011, a criação da Rede de Atenção Psicossocial (RAPS) estabeleceu diretrizes e objetivos para cada ponto da rede, definições estas que colocam em evidência a capacidade resolutiva e operacional esperada para cada instituição que compõe essa rede de serviços ${ }^{1}$.

A satisfação dos usuários reflete os resultados alcançados pelo serviço com seus respectivos determinantes de insatisfação. Esse indicador é capaz de gerar informações para influenciar a tomada de decisão e proporciona oportunidades para os gestores avaliarem aspectos dos CAPS que não se limitem apenas aos aspectos administrativos, mas contemplem a visão dos principais usuários dos serviços ${ }^{3,4}$. A medida subjetiva de satisfação é também um importante componente para participação dos usuários na construção e mudança dos serviços, uma oportunidade para exercerem a cidadania, reforçar o empoderamento (empowerment) sobre seu tratamento, recuperação (recovery) e reabilitação psicossocial ${ }^{5-7}$.

Nos serviços de saúde mental, a satisfação está relacionada à facilidade dos pacientes em aderir ao plano terapêutico, dar continuidade ao tratamento e utilizar o serviço com maior frequência, além de ser um fator que contribui para qualidade de vida e redução das hospitalizações futuras $^{3,4}$. Estudos internacionais que utilizaram escalas de auto relato para avaliação da satisfação dos usuários observaram relação entre a satisfação e variáveis relacionadas às características do próprio paciente, aos profissionais, aspectos sobre o serviço ou evolução do tratamento ${ }^{8-10}$. No Brasil, há um pequeno número de estudos publicados sobre avaliações dos serviços de saúde mental, sobretudo aqueles que descrevem a satisfação dos usuários ${ }^{11}$. Em geral, os usuários encontram-se satisfeitos com os serviços de saúde mental ${ }^{4,6,12-14}$ entretanto, a quantidade de variáveis avaliadas e exploradas nos estudos ainda é reduzida, comparada a outros seguimentos da avaliação. Estudos que estabeleçam comparações relacionadas à modalidade dos CAPS não foram observadas, e podem ser importantes para compreender aspectos de cada modalidade na RAPS de uma região.

Diante da implantação de uma estratégia de atenção à saúde mental, a avaliação dos serviços torna-se necessária, a fim de detectar a resolubilidade dos serviços e capacidade de atender às perspectivas dos usuários, considerando-os como protagonistas na produção de conhecimento ${ }^{7}$. Nesse contexto, o objetivo da pesquisa foi avaliar a satisfação de usuários dos principais CAPS de uma região de Minas Gerais, correlacionando os níveis de satisfação em diferentes modalidades de serviço e características sociodemográficas dos usuários.

\section{Métodos}

Foi realizado um estudo transversal e correlacional, que visa identificar os fatores associados à satisfação de usuários de serviços de Saúde Mental. Os dados foram obtidos por meio da aplicação de uma escala validada para aferir a satisfação dos usuários de serviços de saúde mental e de um formulário semi-estruturado, desenvolvido para a coleta de dados sociodemográficos dos usuários e informações sobre a terapia medicamentosa.

A satisfação dos usuários foi mensurada com a aplicação da versão abreviada da Escala de Avaliação da Satisfação dos Usuários com os Serviços de Saúde Mental (SATIS-BR). A Escala SATIS-BR foi elaborada pela Divisão de Saúde Mental da Organização Mundial de Saúde (OMS) e, posteriormente, validada para uso no Brasil ${ }^{3}$. Essa avaliação está organizada em três subescalas que dimensionam a satisfação por meio de uma escala do tipo likert (pontuação 1 - muito insatis- 
feito - a pontuação 5- muito satisfeito) quanto à competência e compreensão da equipe, acolhida da equipe e ajuda recebida, condições físicas e conforto do serviço avaliado.

O formulário semi-estruturado incluiu as variáveis sociodemográficas presentes na escala abreviada SATIS e foi construído com o objetivo de identificar o perfil dos usuários do serviço e alguns aspectos sobre a terapia medicamentosa. O formulário também incluiu variáveis como o nível de instrução, qualificação e tempo de tratamento no CAPS. Variáveis apontadas por profissionais do serviço em um teste piloto, realizado em dois CAPS, resultou na inclusão das variáveis custo e tempo de deslocamento até o CAPS. Estas variáveis foram sugeridas como fatores importantes para permanência e continuidade do tratamento dos usuários nos CAPS, conforme experiência e relato dos profissionais que atuam nestes serviços.

As informações sobre os medicamentos em uso foram coletadas diretamente das prescrições ou prontuários dos usuários pelos pesquisadores e o formulário incluiu variáveis relacionadas à terapia: descrição dos medicamentos em uso, relato sobre o uso inadequado e busca por orientação sobre o uso dos medicamentos.

Foi elaborado um roteiro de aplicação da pesquisa para o treinamento dos pesquisadores envolvidos na coleta de dados, bem como instruções para padronizar a abordagem dos usuários e aplicação do formulário de pesquisa e da escala SATIS. A relação de usuários presentes no serviço e aptos para participação na pesquisa foi atualizada em todos os dias de coleta dos dados de forma a evitar abordagem de usuários que não estivessem em condição de participar e identificar novos usuários acolhidos nos serviços.

O cálculo amostral para determinação do número mínimo de entrevistados considerou a população das cidades de Betim-MG (417 mil habitantes), Contagem-MG (648 mil habitantes) e Ibirité-MG (173 mil habitantes), no ano de 2014. O cálculo considerou prevalência a priori de 50\%, devido à heterogeneidade dos eventos avaliados, erro de $5 \%$, intervalo de confiança de $95 \%$ e $30 \%$ de perdas. O número mínimo de entrevistas aplicadas em cada CAPS considerou a razão entre a população da cidade em que a instituição estava alocada e a população total das cidades inclusas na pesquisa, dividindo-se esse valor pelo número de CAPS presentes nestas cidades. Utilizou-se o software OpenEpi ${ }^{\circledR}$ versão 3.02 para realizar o cálculo amostral, o qual obteve o valor mínimo de 500 participantes.
A coleta de dados foi realizada nos 11 CAPS de médio e grande porte, sendo dois CAPS II, quatro CAPS III, dois CAPSi, um CAPS ad e dois CAPS ad III. Todos os gestores autorizaram a participação da instituição por meio de envio do termo de anuência à pesquisa. $\mathrm{O}$ período de recrutamento dos participantes ocorreu entre agosto de 2014 e fevereiro de 2015, a coleta de dados em cada CAPS durou em média 30 dias e ocorreu de forma simultânea em algumas instituições de localidades próximas. As entrevistas foram realizadas em datas pré-definidas pelos gestores dos serviços, que prezaram pela disponibilidade do suporte necessário à equipe de coleta da pesquisa e acesso aos CAPS nos dias com oferta de várias atividades, caracterizando funcionamento típico do estabelecimento. A programação das datas de coleta de dados e respectivos locais considerou a alternância dos dias em função da alteração da equipe de profissionais em turnos ou plantões, a fim de abordar usuários que frequentavam os serviços em dias diferenciados para realizar consultas de retorno e rotina. As entrevistas foram conduzidas em locais reservados ou abertos, que garantissem a conversa do participante e seu responsável (quando presente) com o entrevistador, sem interferência de outros usuários ou funcionários dos CAPS. Todos os dados sobre o uso de medicamentos foram confirmados pelos pesquisadores no prontuário do paciente ou prescrição médica mais atual arquivada no serviço ou que estivesse em posse do usuário após a realização da entrevista.

Os participantes do estudo compreenderam uma amostra sistemática de usuários que realizavam tratamento no serviço, em regime intensivo ou não, nos dias preestabelecidos para coleta de dados. O critério de inclusão dos usuários em regime de internação no serviço foi a indicação pelos profissionais. A equipe identificava os usuários em condições de realizar a entrevista no momento, ou seja, pacientes que não estavam em crises agudas nos últimos dias. Os usuários foram convidados a participar da pesquisa mediante assinatura do Termo de Consentimento Livre e Esclarecido (TCLE) da pesquisa. Familiares e/ ou responsáveis por acompanhar os usuários poderiam assinar o TCLE e participar concomitantemente na resposta aos formulários repassando informações. Durante a entrevista foram excluídos do estudo usuários que não compreendiam as perguntas da escala SATIS, principal instrumento da pesquisa, mesmo após a explicação do pesquisador, caracterizando algum quadro de confusão mental. Também foram excluídos 
da pesquisa usuários que apresentaram inquietação ou distorção das informações declaradas, demonstrando não haver condições psicológicas de participação. A elaboração do banco de dados incluiu métodos de controle de qualidade, houve dupla digitação de $10 \%$ dos dados no qual observou-se excelente concordância $(k>0,80)$ entre os dois digitadores. A discordância das informações durante essa conferência motivou a revisão dos dados por ambos os pesquisadores. A concordância inter observador para os dados coletados em prontuários e prescrições médicas foi substancial $(\mathrm{k}=0,79 ; \mathrm{p}=0,00)$, indicando pouca divergência na extração dos dados. A concordância se faz necessária para este quesito, pois nenhum dos serviços apresentou prontuário eletrônico e a extração dos dados foi realizada mediante a leitura dos documentos manuscritos.

A descrição de características sociodemográficas, clínicas e a avaliação dos escores de satisfação por subescalas e por itens foram realizadas por medidas de tendência central e de dispersão, assim como por porcentagens. Foram calculadas as porcentagens de usuários que declararam estar muito satisfeitos ou satisfeitos, cujos escores atingiram a pontuação entre 4 e 5 ; usuários mais ou menos satisfeitos, para respostas com pontuação 3 , e aqueles insatisfeitos com os serviços de saúde mental, com pontuação 1 ou 2 para as respostas. As análises dos dados foram realizadas por meio do software Statistical Package for the Social Sciences (SPSS), versão 19.0. Foram utilizados testes não paramétricos, conforme indicação dos testes de homogeneidade Kolmogorov - Smirnov $(0,21 ; \mathrm{p}=0,00)$ e Shapiro - Wilk $(0,79 ; \mathrm{p}=0,00)$.

Utilizou-se o teste não paramétrico de kendall's para comparação das médias para as três subescalas da SATIS-BR, a fim de identificar se havia diferenças significativas entre seus valores; o teste de Wilcoxon comparou os valores das médias das subescalas duas a duas, com o objetivo de identificar quais pares das subescalas apresentavam diferenças significativas; e o teste de Friedman foi utilizado para identificar itens específicos na escala que apresentavam média de postos mais elevados ou mais baixos de satisfação, ou seja, o objetivo do teste foi identificar na escala aquelas perguntas que sobressaíram por apresentarem postos muito acima ou inferiores aos obtidos nas demais perguntas. Foi adotado um nível de significância de 5\% para todas as análises. Estes testes buscaram comparar os valores obtidos de diferentes maneiras a fim de estabelecer quais itens da escala SATIS se destacam na mensuração da satisfação dos usuários.
Para avaliar a correlação entre o grau de satisfação dos usuários e características sociodemográficas ou clínicas foi realizada uma análise estatística univariada. As variáveis foram categorizadas e avaliadas pelo teste Man-Whitney para comparação dos grupos, considerando o nível de significância de $5 \%$. O teste de Man-Whitney verifica a associação entre duas variávéis não paramétricas ao comparar grupos com diferentes indivíduos. Assim grupos de usuários foram estabelecidos para comparar o nível de satisfação obtido em cada um deles e identificar variações significativas. Uma análise de regressão não pode ser realizada visto que os dados dos escores de satisfação não apresentaram distribuição normal.

As perguntas 13 a 15 da escala SATIS-BR são opcionais e visam registrar considerações que não tenham sido contempladas pelas questões da escala, descrevendo a opinião do usuário sobre o que era melhor ou pior no serviço e o que deveria ser melhorado. A análise destes dados foi realizada pela categorização das respostas em assuntos mais frequentes, mediante a análise de cada uma das respostas. Quando a resposta abordava mais de um assunto foi considerado aquele mais relevante para o usuário ou o primeiro a ser citado/ lembrado, mediante confirmação da relevância/ ordem de prioridade designada pelo usuário quando questionado pelo pesquisador durante a aplicação do formulário de pesquisa.

O estudo foi aprovado pelo Comitê de Ética em Pesquisa da Universidade Federal de Minas Gerais.

\section{Resultados}

Foram realizadas 467 entrevistas válidas em todos os 11 CAPS inclusos na pesquisa. As instituições estão localizadas nas três cidades mais populosas da Região do Médio Paraopeba-MG, sendo serviços de referência para uma população de mais de um 1,2 milhões de habitantes.

Os usuários dos CAPS eram em sua maioria do sexo masculino $(63,17 \%)$, solteiros $(67,75 \%)$, com faixa etária predominante entre 25 a 59 anos $(65,59 \%)$, a idade média dos participantes foi de 34,77 anos $(\mathrm{DP}=16,54)$. A escolaridade concentrava-se nos anos iniciais do ensino fundamental $(51,49 \%)$, com muitos deles exercendo ocupações de nível fundamental ou profissionalizante $(34,74 \%)$ (Tabela 1).

Os usuários entrevistados realizavam consultas com os próprios especialistas dos CAPS dos quais frequentavam $(98,29 \%)$ e a maioria 
Tabela 1. Características sociodemográficas de usuários dos CAPS da Região do Médio Paraopeba-MG, 20142015.

\begin{tabular}{|c|c|c|}
\hline Variáveis & Categorias & Frequência $(\%)$ \\
\hline Idade & 0 a 4 anos & $2(0,43 \%)$ \\
\hline \multirow[t]{7}{*}{$(n=465)$} & 5 a 9 anos & $40(8,60 \%)$ \\
\hline & 10 a 14 anos & $29(6,24 \%)$ \\
\hline & 15 a 19 anos & $37(7,96 \%)$ \\
\hline & 20 a 24 anos & $25(5,38 \%)$ \\
\hline & 25 a 39 anos & $141(30,32 \%)$ \\
\hline & 40 a 59 anos & $164(35,27 \%)$ \\
\hline & 60 anos ou mais & $27(5,81 \%)$ \\
\hline Gênero & Masculino & $295(63,17 \%)$ \\
\hline$(\mathrm{n}=467)$ & Feminino & $172(36,83 \%)$ \\
\hline Ocupação & Nunca Trabalhou & $18(4,47 \%)$ \\
\hline \multirow[t]{8}{*}{$(\mathrm{n}=403)$} & Funções sem qualificação & $82(20,35 \%)$ \\
\hline & Funções nível fundamental/profissionalizante & $140(34,74 \%)$ \\
\hline & Funções nível médio/técnico & $22(5,46 \%)$ \\
\hline & Estudante & $103(25,56 \%)$ \\
\hline & Funções de nível superior & $9(2,23 \%)$ \\
\hline & Aposentado/benefício social & $21(5,21 \%)$ \\
\hline & Desempregado & $6(1,49 \%)$ \\
\hline & Outros & $2(0,50 \%)$ \\
\hline Estado Civil & Casado/união estável/mora com companheiro (a) & $96(20,78 \%)$ \\
\hline \multirow[t]{2}{*}{$(\mathrm{n}=462)$} & Solteiro & $313(67,75 \%)$ \\
\hline & Divorciado/separado & $53(11,47 \%)$ \\
\hline Escolaridade & Nunca estudou & $10(2,48 \%)$ \\
\hline \multirow[t]{9}{*}{$(\mathrm{n}=404)$} & Escola especial & $2(0,50 \%)$ \\
\hline & Fundamental incompleto & $208(51,49 \%)$ \\
\hline & Fundamental completo & $47(11,63 \%)$ \\
\hline & Ensino médio incompleto & $46(11,39)$ \\
\hline & Ensino Médio completo & $72(17,82 \%)$ \\
\hline & Ensino médio Técnico & $7(1,73 \%)$ \\
\hline & Superior incompleto & $5(1,24 \%)$ \\
\hline & Superior completo & $6(1,49 \%)$ \\
\hline & Doutorado & $1(0,25 \%)$ \\
\hline
\end{tabular}

era responsável pela busca da medicação que fazia uso $(70,63 \%)$. Mais da metade dos usuários estavam em tratamento e vinculados ao serviço por até dois anos (59,77\%). Muitos usuários dos CAPS não possuíam custos com deslocamento $(60,09 \%)$ em função de utilizar o transporte oferecido pela instituição ou por morar nas proximidades dos CAPS. Grande parte dos usuários $(75,47 \%)$ demoravam até 40 minutos para chegar ao serviço de saúde mental (Tabela 2).

Os usuários dos CAPS utilizavam em média 3,38 ( $\mathrm{DP}=1,76)$ medicamentos durante o dia, variando entre 0 a 9 medicamentos. Quando questionados sobre os medicamentos em uso, $45,80 \%$ dos usuários sabiam dizer ou ler o nome de todos medicamentos registrados em suas prescrições e/ou prontuários; 39,23\% não tinham conhecimento sobre o nome de nenhum deles. Um terço dos participantes $(34,78 \%)$ declararam que já fizeram uso inadequado de medicamentos em algum momento de seu tratamento e 73,14\% afirmaram que procuram tirar dúvidas sobre o uso de medicamentos. O médico foi o profissional mais citado para esclarecer dúvidas sobre os medicamentos $(48,40 \%)$, seguido do enfermeiro $(8,40 \%)$ e farmacêutico $(7,82 \%)$. Destaca-se que muitos usuários consultam outros profissionais de saúde ou mesmo pessoas próximas como familiares e amigos (13,30\%) para sanar dúvidas sobre o uso de medicamentos. 


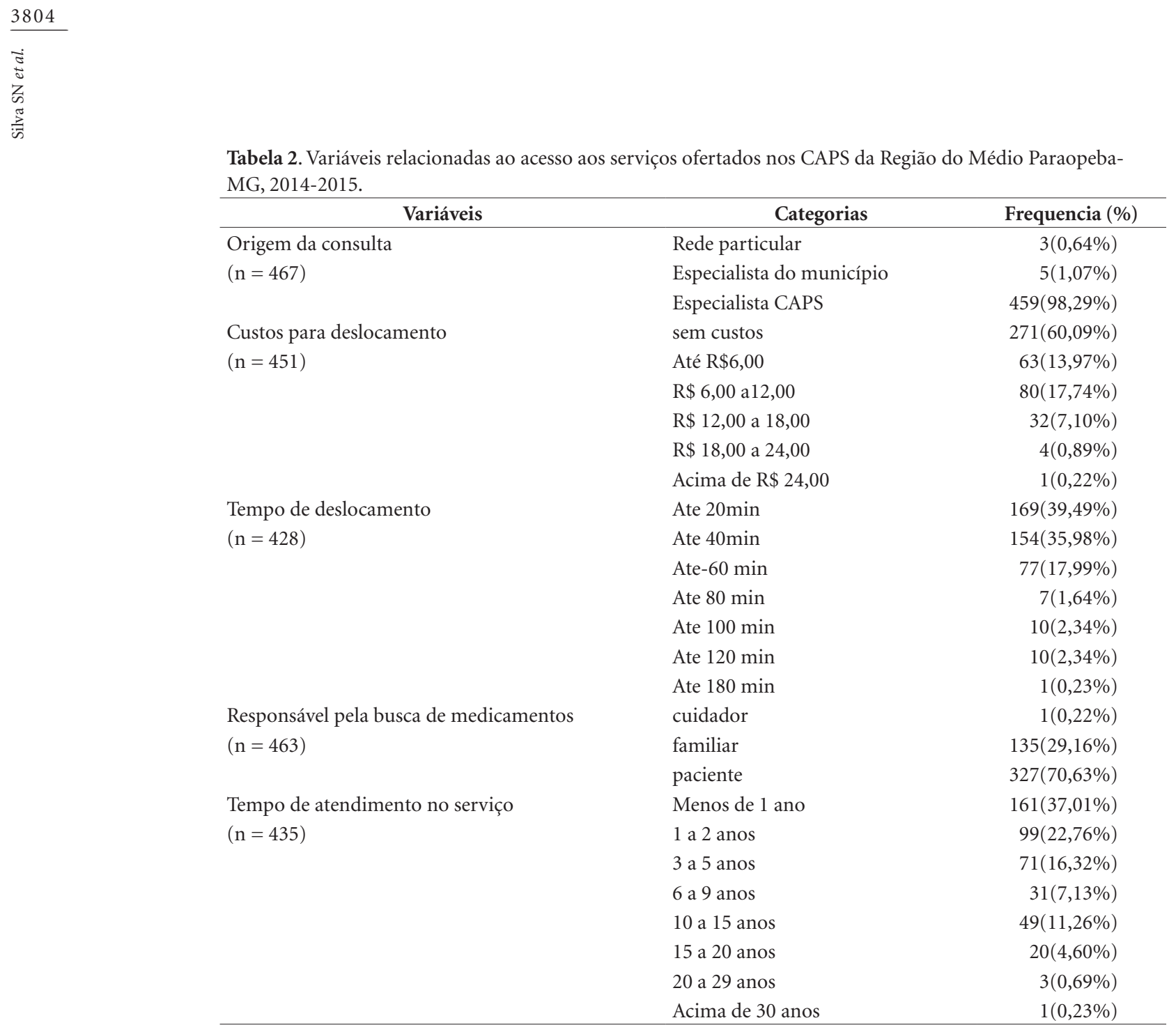

A análise de satisfação dos usuários dos serviços foi apresentada por subescalas e por uma escala global. O escore médio de satisfação global dos usuários foi de 4,46 (DP = 0,65) em uma escala que varia de 1 a 5 , indicando que os usuários encontravam-se satisfeitos ou muito satisfeitos com o serviço. As subescalas também apresentaram escores elevados: a subescala 1 , relacionada à competência e compreensão da equipe, apresentou média de $4,54(\mathrm{DP}=0,65)$; a subescala 2 , relacionada à acolhida da equipe e ajuda recebida, apresentou a maior média, de 4,64 $(\mathrm{DP}=0,71)$; e, a subescala 3 , que relaciona a satisfação às condições físicas e conforto do serviço, apresentou a menor média, de 4,09 (DP = 1,06) (Tabela 3).

A comparação das médias das subescalas, pelo teste kendall's, indicou diferença significativa entre elas $\left(X^{2}=206,77\right)$. As comparações das médias das subescalas por pares, realizado por meio do teste não paramétrico de Wilcoxon, con- firmou que os usuários estão mais satisfeitos com a acolhida da equipe e ajuda recebida, comparado à competência e compreensão da equipe e a satisfação com as condições físicas e conforto do serviço (Tabela 3).

A análise de cada item da escala SATIS-BR mostra a porcentagem de usuários satisfeitos ou muito satisfeitos, usuários com satisfação mediana; e usuários insatisfeitos (Tabela 4). Em todos os itens da escala, a maioria dos usuários demonstrou estar satisfeita ou muito satisfeita. As perguntas relacionadas à satisfação com a competência da pessoa com quem trabalhou mais próximo, satisfação com a acolhida dos profissionais e satisfação com a maneira como foi tratado em termos de respeito e dignidade destacaram-se como as porcentagens mais elevadas. Os itens satisfação com o conforto e a aparência do serviço e satisfação com as condições gerais das instalações alcançaram as menores porcentagens. 
Tabela 3. Média de satisfação dos pacientes com o serviço e comparação das médias das subescalas, duas a duas, pelo teste não paramétrico de Wilcoxon nos CAPS da Região do Médio Paraopeba-MG, 2014-2015.

\begin{tabular}{|c|c|c|c|c|}
\hline Subescalas & Média (DP) & $\mathrm{p}(1-2)$ & $\begin{array}{l}\text { Wilcoxon } \\
\mathrm{p}(1-3)\end{array}$ & $\mathrm{p}(2-3)$ \\
\hline 1. Satisfação com a competência e compreensão da equipe & $4,54(0,65)$ & & & \\
\hline 2. Satisfação com a acolhida da equipe e ajuda recebida & $4,64(0,71)$ & $0,00^{\star}$ & & $0,00^{*}$ \\
\hline 3.Satisfação com as condições físicas e conforto do serviço & $4,09(1,06)$ & & $0,00^{*}$ & \\
\hline Escala global & $4,46(0,65)$ & & & \\
\hline
\end{tabular}

Tabela 4. Porcentagem de respostas dos pacientes para cada item da escala SATIS-BR e médias de postos de friedman, nos CAPS da região do Médio Paraopeba-MG, 2014-2015.

\begin{tabular}{|c|c|c|c|c|c|}
\hline Perguntas escala SATIS-BR reduzida & $\mathbf{N}$ & $\begin{array}{c}\text { Insatisfeito } \\
\text { (Pontuação } 1 \\
\text { e } 2 \text { na escala) }\end{array}$ & $\begin{array}{c}\text { Intermediário } \\
\text { (Pontuação } 3 \\
\text { na escala) }\end{array}$ & $\begin{array}{c}\text { Satisfeito } \\
\text { (Pontuação } 4 \\
\text { e } 5 \text { na escala) }\end{array}$ & $\begin{array}{c}\text { média } \\
\text { de } \\
\text { postos }\end{array}$ \\
\hline \multicolumn{6}{|l|}{$\begin{array}{l}\text { Subescala } 1 \text { - Satisfação com a competência e } \\
\text { compreensão da equipe }\end{array}$} \\
\hline Pessoa que o admitiu no serviço & 457 & $25(5,47 \%)$ & $37(8,10 \%)$ & $395(86,43 \%)$ & 6,92 \\
\hline Compreensão do seu problema na admissão & 451 & $33(7,32 \%)$ & $37(8,20 \%)$ & $381(84,48 \%)$ & 6,58 \\
\hline $\begin{array}{l}\text { Compreensão da equipe sobre o tipo de } \\
\text { ajuda que necessitava }\end{array}$ & 456 & $41(8,99 \%)$ & $32(7,01 \%)$ & $383(84,00 \%)$ & 6,33 \\
\hline Tipo de ajuda ofertada pelo serviço & 454 & $21(4,62 \%)$ & $36(7,93 \%)$ & $397(87,45 \%)$ & 6,58 \\
\hline Discussão do seu tratamento & 452 & $30(6,64 \%)$ & $37(8,18 \%)$ & $385(85,18 \%)$ & 6,16 \\
\hline Competência da equipe do serviço & 453 & $17(3,75 \%)$ & $38(8,39 \%)$ & $398(87,86 \%)$ & 6,72 \\
\hline $\begin{array}{l}\text { Competência da pessoa com quem você } \\
\text { trabalhou mais próximo }\end{array}$ & 450 & $9(2,00 \%)$ & $16(3,56 \%)$ & $425(94,44 \%)$ & 7,40 \\
\hline \multicolumn{6}{|l|}{$\begin{array}{l}\text { Subescala } 2 \text { - Satisfação com a acolhida da } \\
\text { equipe e ajuda recebida }\end{array}$} \\
\hline $\begin{array}{l}\text { Maneira como foi tratado em termos de } \\
\text { respeito e dignidade? }\end{array}$ & 462 & $23(4,98 \%)$ & $25(5,41 \%)$ & $414(89,61 \%)$ & 6,94 \\
\hline Ajuda da equipe & 455 & $17(3,74 \%)$ & $33(7,25 \%)$ & $405(89,01 \%)$ & 7,10 \\
\hline Acolhida dos profissionais & 458 & $16(3,49 \%)$ & $31(6,77 \%)$ & $411(89,74 \%)$ & 6,87 \\
\hline \multicolumn{6}{|l|}{$\begin{array}{l}\text { Subescala } 3 \text { - Satisfação com as condições } \\
\text { físicas e conforto do serviço }\end{array}$} \\
\hline Conforto e a aparência do serviço & 459 & $52(11,33 \%)$ & $55(11,98 \%)$ & $352(76,69 \%)$ & 5,52 \\
\hline Condições gerais das instalações & 450 & $58(12,89 \%)$ & $77(17,11 \%)$ & $315(70,00 \%)$ & 4,88 \\
\hline
\end{tabular}

*teste de friedman $\mathrm{X}^{2}=393,49 \mathrm{p}=0,000$.

A análise da média de postos de Friedman para cada item da escala de satisfação apontou uma diferença significativa entre os 12 itens da escala $\left(X^{2}=393,49 ; \mathrm{p}=0,000\right)$. As médias mais elevadas correspondem às perguntas sobre a satisfação com a competência da pessoa com quem você trabalhou mais próximo, satisfação com a ajuda da equipe e satisfação com a pessoa que o admitiu no serviço.

As perguntas abertas, destinadas a coletar a opinião adicional dos usuários sobre o serviço, foram categorizadas e agrupadas em temas mais freqüentes. Mais de metade dos participantes (n =252) demonstrou interesse em registrar o que consideravam melhor no serviço. As respostas mais frequentes fizeram referência à acolhida ao serviço, ao convívio e respeito entre profissionais e usuários (35,31\%), elogios às oficinas e atividades desenvolvidas nos CAPS (26,59\%) e elogios aos profissionais $(25,40 \%)$. Foram registradas 101 contribuições sobre os piores aspectos dos serviços, das quais $28,71 \%$ fizeram referência 
à infraestrutura precária. Dentre as 218 contribuições com sugestões de melhorias apontadas pelos usuários, destacam-se melhorias na infraestrutura $(55,04 \%)$, necessidade de aumento do número de profissionais alocados nos serviços $(11,93 \%)$ e implantação de mais atividades e oficinas $(9,63 \%)$.

A análise univariada analisou a associação entre as variáveis sociodemográficas e clínicas e o nível de satisfação dos usuários. Observou-se diferença com significância estatística somente para as variáveis trabalho, porte e modalidade do serviço e tempo de atendimento (Tabela 5). Dessa forma, os usuários que trabalhavam, assim como os que realizavam tratamento no serviço a menos de dois anos apresentaram maior nível de satisfação comparados àqueles que não exerciam atividade profissional ou estavam em tratamento há mais de 2 anos nos serviços. Os usuários atendidos em serviços de menor porte, os CAPS II, estavam mais satisfeitos que aqueles atendidos em serviços de grande porte com funcionamento de 24 horas- CAPS III. Usuários atendidos em serviços de saúde mental estavam menos satisfei- tos que aqueles atendidos em serviços exclusivos para o tratamento de dependência em álcool e outras drogas, os CAPS ad.

\section{Discussão}

Os resultados sobre a satisfação dos usuários indicaram que a grande maioria está satisfeita ou muito satisfeita com os CAPS da Região do Médio Paraopeba-MG, resultados estes coerentes com outros estudos de satisfação realizados pelo Brasil utilizando a escala SATIS-BR ${ }^{6,12,15,16}$. Estudos internacionais indicam, em geral, satisfação moderada a boa dos usuários dos serviços de saúde mental, níveis mais modestos que os encontrados em estudos brasileiros ${ }^{17}$.

O perfil demográfico dos usuários entrevistados destaca como maioria os homens solteiros, adultos na faixa etária economicamente ativa, que apresentam poucos anos de escolaridade e consequentemente desempenhavam atividades profissionais de baixa qualificação. Este perfil demográfico difere de muitos estudos por apre-

Tabela 5. Análise univariada de variáveis categóricas sociodemográficas e dos pacientes e dos serviços associadas à satisfação global dos pacientes, pelo teste de Man-Whitney nos CAPS da região do Médio Paraopeba-MG, 2014-2015.

\begin{tabular}{|c|c|c|c|c|}
\hline Variáveis & Categorias & $\mathbf{N}$ & Média (DP) & $\mathbf{P}$ \\
\hline \multirow[t]{2}{*}{ Gênero } & Feminino & 262 & $4,47(0,62)$ & 0,97 \\
\hline & Masculino & 150 & $4,44(0,69)$ & \\
\hline \multirow[t]{2}{*}{ Idade } & Faixa etária economicamente inativa & 85 & $4,51(0,58)$ & 0,61 \\
\hline & Faixa etária economicamente ativa ( 15 a 60$)$ anos & 326 & $4,44(0,66)$ & \\
\hline \multirow[t]{2}{*}{ Trabalho } & Não trabalha & 134 & $4,41(0,66)$ & $0,04^{*}$ \\
\hline & Trabalha & 228 & $4,51(0,62)$ & \\
\hline \multirow[t]{2}{*}{ Escolaridade } & Ensino médio incompleto & 277 & $4,53(0,57)$ & 0,90 \\
\hline & Ensino médio completo & 81 & $4,23(0,83)$ & \\
\hline \multirow[t]{2}{*}{ Estado Civil } & Casado/união estável & 120 & $4,40(0,71)$ & 0,90 \\
\hline & Solteiro/divorciado/viúvo & 267 & $4,48(0,63)$ & \\
\hline \multirow[t]{2}{*}{ Medicamentos } & Usa até 4 medicamentos prescritos para saúde mental & 267 & $4,48(0,63)$ & 0,24 \\
\hline & Usa 5 ou mais medicamentos para saúde mental & 120 & $4,39(0,71)$ & \\
\hline \multirow[t]{2}{*}{ Porte do serviço } & Paciente atendido em serviços de menor porte (CAPS II) & 180 & $4,56(0,52)$ & $0,02^{\star *}$ \\
\hline & Paciente atendido em serviços 24horas (CAPS III) & 231 & $4,38(0,72)$ & \\
\hline \multirow[t]{2}{*}{ Modalidade de serviço } & Pacientes serviços de saúde mental & 313 & $4,38(0,68)$ & $0,00^{\star * \star}$ \\
\hline & Pacientes serviços de álcool e outras drogas CAPSad & 98 & $4,72(0,43)$ & \\
\hline \multirow[t]{2}{*}{ Gasto deslocamento } & Não há gastos do pacientes para chegar até o CAPS & 248 & $4,43(0,67)$ & 0,15 \\
\hline & há gastos para o paciente chegar até ao CAPS & 155 & $4,52(0,60)$ & \\
\hline \multirow[t]{2}{*}{ Tempo deslocamento } & Gasta até 40 minutos para deslocar ate o CAPS & 289 & $4,45(0,66)$ & 0,33 \\
\hline & Gasta mais de 40 minutos para deslocar até o CAPS & 95 & $4,50(0,61)$ & \\
\hline \multirow[t]{2}{*}{ Tempo de atendimento } & Paciente assistido pelo serviço por até 2 anos & 231 & $4,49(0,65)$ & $0,00 \#$ \\
\hline & Paciente assistido pelo serviço por mais de 2 anos & 152 & $4,36(0,64)$ & \\
\hline
\end{tabular}

${ }^{\star} 0,043 ;{ }^{* *} 0,022 ;{ }^{* * *}<0,001 ; \# 0,002$. 
sentar maior número de usuários do sexo masculino, condição que pode ser justificada pela inclusão dos serviços CAPS ad no estudo. Os usuários do sexo masculino são maioria nos CAPS ad, resultado que acompanha outros estudos e o levantamento sobre o consumo de drogas psicotrópicas $^{15,18}$. Destaca-se que as mulheres possuem um perfil social diferente e enfrentam problemas relacionados ao estigma social na procura pelo tratamento da dependência, o que também pode contribuir para menor frequência no CAPS $\mathrm{ad}^{15}$.

A maioria dos usuários realizavam consultas com os especialistas dos CAPS dos quais frequentavam, retiravam a medicação prescrita no estabelecimento e relataram acesso facilitado ao serviço no que se refere à isenção de custos e baixo tempo de deslocamento. O estudo de Mercier e Corten ${ }^{19}$, já indicava que a satisfação dos usuários de serviços de saúde mental estava relacionada às questões de acesso como a disponibilidade de serviços gratuitos, continuidade dos cuidados e isenção de custos com medicamentos, questões estas que podem contribuir para compreender os altos escores de satisfação dos usuários dos CAPS dessa região. Destaca-se, porém, que a proposta dos CAPS ultrapassa estes critérios, pois além de garantir o acesso, é preciso que os usuários sintam-se acolhidos e satisfeitos, a fim de construir junto com a equipe o PTS, respeitando sua subjetividade e a possibilidade de estabelecer vínculo com o serviço ${ }^{20}$.

Os fatores preditores de satisfação dos serviços de saúde mental devem ser identificados e trabalhados pelo gestor como um importante elemento para efetividade do serviço. A percepção de mudança pelo usuário foi atribuída como principal preditora de satisfação com o serviço por um estudo brasileiro ${ }^{16}$. Já um estudo realizado em um estado norte-americano identificou como fatores preditores o acesso, qualidade e participação do paciente em seu plano terapêutico ${ }^{21}$.

Os usuários encontravam-se satisfeitos com os CAPS e os maiores escores de satisfação observados nesse estudo foram referentes à competência dos profissionais que conduziam o tratamento, acolhida e ajuda recebida no serviço, resultados que corroboram com estudos desenvolvidos em outras regiões do país ${ }^{6,12,15,16}$. Um estudo realizado em um Hospital psiquiátrico da Inglaterra avaliou a importância das relações no cuidado em saúde mental. Os relatos dos pacientes relacionados a experiência no serviço, faziam referência muitas vezes aos profissionais que tiveram contato na sua admissão, ressaltando a importância destas relações durante a hospitalização ${ }^{22}$.
A satisfação é um constructo multidimensional formulado principalmente pelo modelo da expectativa, pelo qual o usuário realiza um julgamento a partir da comparação entre a expectativa prévia e a sua real experiência com o serviço ${ }^{23}$. Assim os altos escores associados podem ser justificados pela superação das expectativas desses usuários, principalmente com relação à equipe de profissionais. Baixas expectativas podem ter sido construídas com base em experiências negativas acumuladas de outros serviços de saúde, com propostas e modelos diferenciados, ou mesmo em outros CAPS com deficiências nestes quesitos.

A satisfação com as condições físicas e conforto do serviço obtiveram a menor pontuação na escala de avaliação SATIS-BR, neste e em outros estudos publicados no Brasil $1^{5,6,12,16}$. Apesar dos usuários estarem satisfeitos ou muito satisfeitos com o serviço em uma avaliação geral, o relato dos itens que necessitam de melhorias revelou algumas fragilidades na estrutura física dos CAPS. Existe atualmente uma padronização para a construção dos CAPS $^{24}$, entretanto, observa-se muitos problemas de infraestrutura e falta de padronização, como a adaptação dos CAPS em imóveis residenciais, falta de adaptações de acessibilidade e a necessidade de reformas ${ }^{25,26}$, pontos importantes capazes de influenciar no nível de satisfação dos usuário que deveriam ser priorizados pelos gestores.

Neste estudo também foi verificado o conhecimento dos usuários sobre a terapia medicamentosa. Muitos usuários não sabiam o nome de nenhum dos medicamentos em uso, o que pode contribuir para o uso inadequado de medicamentos, uma questão relatada pelos próprios usuários. Vale ressaltar que grande parte dos usuários está satisfeita com o serviço, mesmo sem conhecer aspectos sobre sua terapia. Em um serviço do Reino Unido os usuários reconheceram a importância do medicamento no tratamento e ressaltaram a importância da comunicação com os usuários e os familiares sobre este assunto. $\mathrm{O}$ uso de medicamentos sem o consentimento do paciente, ou de forma coerciva em serviços de internação, gera experiências negativas que poderão trazer prejuízos à continuidade do tratamento ${ }^{22}$.

Foram identificados neste estudo usuários que utilizavam até nove medicamentos, todos relacionados apenas ao tratamento dos transtornos mentais. Estes usuários estão sujeitos a muitas reações adversas, efeitos colaterais e interações medicamentosas ${ }^{27}$, condições que podem influenciar na efetividade, adesão e continuidade 
do tratamento proposto. Um assunto pertinente nesse contexto é a necessidade de reorganização da Assistência Farmacêutica especializada no atendimento dos serviços de saúde mental e capacitação dos profissionais ${ }^{28,29}$. Especificamente na Região do Médio Paraopeba-MG existe uma organização da assistência farmacêutica adequada mas que pouco atua em práticas assistenciais como a inserção de atividades direcionadas aos usuários para melhor compreensão da terapia medicamentosa $\mathrm{a}^{30}$. Um estudo apontou que a dispensação de medicamentos nos CAPS colaborou para o alto nível de satisfação dos usuários e familiares ${ }^{5}$. No entanto, a relação entre a satisfação dos usuários, o nível de conhecimento e a adesão à terapia representam uma lacuna que necessita de estudos futuros e são questões importantes a serem verificadas nos serviços de saúde mental.

O desconhecimento da terapia medicamentosa é outro fator que transparece a necessidade de reforçar a participação do usuário na construção do seu projeto terapêutico e empoderamento sobre sua terapia. Questões como o gerenciamento da terapia podem inclusive, refletir na sua compreensão e adesão ao tratamento. A introdução da Gestão Autônoma do Medicamento (GAM) é uma prática inovadora que apresenta resultados positivos na qualidade de vida e bem-estar do usuário. Ao compreender os efeitos dos medicamentos e função no tratamento do seu transtorno mental, os usuários são capazes de ampliar a participação, agir de forma mais autônoma em relação à medicação e transformar a relação com os profissionais de saúde ${ }^{31}$. Resultados positivos de experiências internacionais reforçam a necessidade de inserção da GAM nos serviços de saúde mental, pois permitem a expressão subjetiva e o posicionamento do usuário, tornando-o mais ativo e crítico, em consonância com a atual e crescente proposta da atenção psicossocial que reforça a reinserção social, autonomia e o exercício da cidadania ${ }^{31,32}$. A participação dos usuários no planejamento do plano terapêutico já é reconhecida como um prática positiva nos serviços de saúde mental e em expansão, sendo monitorada pelo sistema de saúde inglês (NHS-National Health System) ${ }^{17,33}$. Estas ações no CAPS são importantes para assegurar que os usuários construam expectativas cada vez mais críticas, que incluam noções sobre seus direitos e participação na construção dos serviços, obtendo uma ampla avaliação sob diversos aspectos.

Maiores escores de satisfação foram associados aos usuários dos CAPS II comparados aos CAPS III que são serviços $24 \mathrm{~h}$ e mantém, ainda que provisório, o acolhimento em período diurno e noturno do usuário por alguns dias. Uma hipótese a ser considerada, nesse caso, é que a privação da liberdade, mesmo que por um período bem delimitado e justificado clinicamente, pode não corresponder à expectativa dos usuários. No momento de lucidez, como na entrevista, o usuário pode não ser capaz de recordar sua condição na crise, fato que justificaria essa tomada de decisão pelos profissionais. Conforme esperado, serviços com propostas e intervenções diferenciadas, tendem a provocar diferentes níveis de satisfação aos usuários ${ }^{16}$. Estudos futuros, mais específicos, poderão indicar se esta diferença de satisfação está atribuída às características da modalidade do serviço ofertado, ao olhar diferenciado do usuário sobre a equipe e o serviço observado por maior período, ou mesmo uma opinião pessoal do usuário. A mesma observação deverá ser realizada para justificar menores escores de satisfação associados aos usuários dos serviços de saúde mental comparados aos CAPS ad, haja vista o perfil diferenciado dos usuários de cada serviço e práticas distintas.

A análise realizada no estudo não apontou associação de maiores escores de satisfacão para os usuários dos Capsi (idade até 15 anos) comparado aos usuários das demais faixas etárias. No entanto a idade é um fator importante que estava associada ao nível de satisfação dos usuários no estudo conduzido por Silva et al., no qual pacientes com maior idade estavam mais satisfeitos com os serviços ${ }^{16}$. Estudos que utilizam avaliações estratificadas por idade devem ser conduzidos a fim de identificar a existência de algum tipo de associação, visto que uma abordagem diferenciada pode ser uma estratégia para estabelecer vínculo do usuário com o serviço.

Maiores escores de satisfação atribuídos por usuários que exercem alguma atividade profissional, ou que estão agregados ao serviço por menor período, também podem refletir diferenças nos níveis de expectativa criadas por cada perfil de usuário. $\mathrm{O}$ reconhecimento dos profissionais dos CAPS, sob a ótica de usuários que também trabalham, pode ser um critério para valorizar ainda mais suas ações. Os usuários que permanecem por mais tempo no serviço, precisam lidar com a aceitação do transtorno mental crônico e conflitos capazes de interferir no nível de satisfação com serviço e também seu tratamento.

Importante considerar que altos valores de satisfação podem refletir a familiaridade do usuário com o serviço, sua gratidão e, principalmente, a valorização de um modelo de atenção 
que difere bastante do tratamento nos hospitais psiquiátricos. O risco de viés de gratidão ocorre quando os usuários omitem as críticas ou tem grande afinidade com os profissionais que lhe atendem ${ }^{34}$, dessa forma pode interferir nos resultados deste e outros estudos com a temática de avaliação de serviços. A análise dos questionamentos registrados pelos usuários nas perguntas abertas da escala SATIS-BR e questões sobre a terapia foram importantes para identificar fragilidades na infraestrutura dos CAPS e desconhecimento dos usuários sobre aspectos da terapia, resultados que convidam à reflexão e investigação da percepção de satisfação que este perfil de usuário pode apresentar.

O estudo apresentou limitações ao estudar uma amostra sem seleção aleatória, necessitando de cautela na generalização dos dados e associações encontradas. Por se tratar de um estudo transversal, não é possível acompanhar a evolução da satisfação em momentos distintos ao longo do tempo. Estudos longitudinais serão capazes de acompanhar a evolução da opinião dos usuá- rios, alterações dos serviços e até mesmo mudança de critérios e exigências impostas pelos usuários. Apesar disso, o estudo abrangeu usuários de diversas modalidades de CAPS e representa um dado importante visto a ausência de avaliações dos CAPS, serviços consolidados recentemente que representaram uma ruptura com os métodos de assistência à saúde mental.

A participação dos usuários na avaliação de serviços de saúde mental é uma prática frequente em países como o Reino Unido, Canadá e Austrália e um tema ainda em construção no Brasil ${ }^{7}$. Utilizar a opinião dos usuários como um critério de avaliação representa uma mudança de paradigma na saúde mental, em um sistema que há poucos anos subestimava sua percepção. A prática de avaliação de serviços de saúde necessita de diversas abordagens que perpassam pela aceitação dos serviços pelos usuários, bem como o cumprimento de critérios técnicos. Assim diferentes estratégias de avaliação devem ser utilizadas para complementar esse complexo processo de avaliação dos serviços de saúde mental.

\section{Colaboradores}

SN Silva e MG Lima contribuíram na concepção, análise de dados e redação do manuscrito, CMR Brandão contribuiu na redação do manuscrito e revisão do conteúdo final.

\section{Referências}

1. Brasil. Ministério da Saúde (MS). Gabinete do Ministro. Portaria $n^{\circ} 3.088$, de 23 de dezembro de 2011. Institui a Rede de Atenção Psicossocial para pessoas com sofrimento ou transtorno mental e com necessidades decorrentes do uso de crack, álcool e outras drogas, no âmbito do Sistema Único de Saúde (SUS). Diário Oficial da União 2013; 21 maio.

2. Brasil. Portaria no 336, de 19 defevereiro de 2002. Define e estabelece diretrizes para o funcionamento dos Centros de Atenção Psicossocial. Diário Oficial da União 2002; 20 fev.

3. Bandeira M, Silva MA. Escala de Satisfação dos Pacientes com os Serviços. J Bras Psiquiatr 2012; 61(3):124132.

4. Silva M. Satisfação dos usuários com os serviços de saúde mental. In: M Bandeira, LA Lima, S Barroso, organizadores. Avaliação de serviços de saúde mental: Princípios metodológicos, indicadores de qualidade e instrumentos de medida. Petrópolis: Vozes; 2014. p. 55-86

5. Miranda PO, Souza OF, Ferreira TF. Avaliação da satisfação dos pacientes e familiares em um serviço de saúde mental na cidade de Rio Branco, Acre. J Bras Psiquiatr 2014; 63(4):332-340. 
6. Kantorski LP, da Rosa Jardim V, Wetzel C, Olschowsky A, Schneider JF, Heck RM, Bielemann VL, Schwartz E, Coimbra VCC, Quevedo ALA, Saraiva SS. Satisfação dos usuários dos centros de atenção psicossocial da região Sul do Brasil. Rev Saude Publica 2009; 43(Supl. 1):29-35.

7. Presotto RF, Silveira M, Delgado PGG, Vasconcelos EM. Experiências brasileiras sobre participação de usuários e familiares na pesquisa em saúde mental. Cien Saude Colet 2013; 18(19):2837-2845.

8. Lunnen KM, Ogles BM, Pappas LN. A multiperspective comparison of satisfaction, symptomatic change, perceived change, and end-point functioning. Professional Psychology: Research and Practice 2008; 39(2):145.

9. Hackman A, Brown C, Yang Y, Goldberg R, Kreyenbuhl J, Lucksted A, Wohlheiter K, Dixon L. Consumer satisfaction with inpatient psychiatric treatment among persons with severe mental illness. Community Ment Health J 2007; 43(6):551-564.

10. Anderson R, Barbara A, Feldman S. What patients want: A content analysis of key qualities that influence patient satisfaction. J Med Pract Manage 2007; 22(5):255-261.

11. Costa PHA, Basile Colugnati FA, Ronzani TM. Avaliação de serviços em saúde mental no Brasil: revisão sistemática da literatura. Cien Saude Colet 2015; 20(10):3243-3253.

12. Camilo CA, Bandeira M, Leal R, Scalon JD. Avaliação da satisfação e sobrecarga em um serviço de saúde mental. Cad Saúde Colet 2012; 20(1):82-92.

13. Heckert U, Teixeira LS, Trindade AS. Avaliação da satisfação dos usuários do Centro Regional de Referência em Saúde Mental (CRRESAM) da região central de Juiz de Fora, MG. HU rev 2006; 32(1):15-19.

14. Jaegger RC, Guitton AP, Lyrio JM, Santos MM, Freitas RCO, Gonçalves SR, Lima LA, Legay LF. A experiência de morar fora: avaliação da satisfação de usuários em um serviço de saúde mental. Cad Saúde Colet 2004; 12(1):27-39.

15. Barbosa GC, Oliveira MAFd, Moreno V, Padovani CR, Claro HG, Pinho PH. Satisfação de usuários num Centro de Atenção Psicossocial em álcool e outras drogas. Revista Portuguesa de Enfermagem de Saúde Mental 2015(14):31-37.

16. Silva MA, Bandeira M, Scalon JD, Quaglia MAC. Satisfação dos pacientes com os serviços de saúde mental: a percepção de mudanças como preditora. J Bras Psiquiatr 2012; 61(2):64-71.

17. Newman D, O’Reilly P, Lee SH, Kennedy C. Mental health service users' experiences of mental health care: an integrative literature review. J Psychiatr Ment Health Nurs 2015; 22(3):171-182.

18. Carlini EA, Noto AR, Sanchez ZM, Carlini CMA, Locatelli DP, Abeid LR, Amato TC, Opaleye ES, Tondowski CL, Moura YG. VI Levantamento nacional sobre o consumo de drogas psicotrópicas entre estudantes do ensino fundamental e médio das redes pública e privada de ensino nas 27 capitais brasileiras-2010. Brasília: SENAD; 2010.

19. Mercier C, Corten P. Évaluation de la qualité de vie de patients psychotiques. In: Kovess V, editor. Evaluation de la qualité en psychiatrie. Paris: Economica; 1994. p. 263-287.

20. Dutra VFD, Oliveira RMP. Revisão integrativa: as práticas territoriais de cuidado em saúde mental. Aquichan 2015; 15(4):529-540.
21. Sohn M, Barrett H, Talbert J. Predictors of Consumer Satisfaction in Community Mental Health Center Services. Community Ment Health J 2014; 50(8):922-925.

22. Gilburt H, Rose D, Slade M. The importance of relationships in mental health care: A qualitative study of service users' experiences of psychiatric hospital admission in the UK. BMC Health Services Research 2008; $8(1): 1$.

23. Bandeira M, Silva MA, Camilo CA, Felício CM. Satisfação de familiares de pacientes psiquiátricos com os serviços de saúde mental e seus fatores associados. $J$ Bras Psiquiatr 2011; 60(4):284-293.

24. Brasil. Ministério da saúde (MS). Manual de Estrutura Física dos Centros de Atenção Psicossocial e Unidades de Acolhimento: Orientações para Elaboração de Projetos de Construção de CAPS e de UA como lugares da Atenção Psicossocial nos territórios. Brasília: MS; 2013.

25. Nascimento AF, Galvanese ATC. Evaluation of psychosocial healthcare services in the city of Sao Paulo, Southeastern Brazil. Rev Saude Publica 2009; 43(Supl. 1):8-15.

26. Silva SN, Lima MG. Avaliação da estrutura dos Centros de Atenção Psicossocial da Região do Médio Paraopeba-Minas Gerais. Epidemiol. Serv. Saúde 2017; 26(1):149-160.

27. Moura CS, Ribeiro AQ, Starling SM. Avaliação de interações medicamentosas potenciais em prescrições médicas do Hospital das clínicas da Universidade Federal de Minas Gerais. Latin american journal of pharmacy 2007; 26(4):596-601.

28. Alencar TOS, Cavalcante EAB, Alencar BR. Assistência farmacêutica e saúde mental no Sistema Único de Saúde. Revista de Ciências Farmacêuticas Básica e Aplicada 2013; 33(4):489-495.

29. Zanella CG, Aguiar PM, Storpirtis S. Atuação do farmacêutico na dispensação de medicamentos em Centros de Atenção Psicossocial Adulto no Município de São Paulo, SP. Cien Saude Colet 2015; 20(2):325-332.

30. Silva SN, Lima, MG. Assistência Farmacêutica na Saúde Mental: um diagnóstico dos Centros de Atenção Psicossocial. Cien Saude Colet 2017; 22(6):2025-2036.

31. del Barrio LR, Cyr C, Benisty L, Richard P. Autonomous Medication Management (GAM): new perspectives on well-being, quality of life and psychiatric medication. Cien Saude Colet 2013; 18(10):2879-2887.

32. Vasconcelos EM. Empoderamento de usuários e familiares em saúde mental e em pesquisa avaliativa/ interventiva: uma breve comparação entre a tradição anglo-saxônica e a experiência brasileira. Cien Saude Colet 2013; 18(10):2825-2835.

33. Care Quality Commission (CQC). National summary of the results for the community mental health survey 2012. [acessado 2016 Set 05]. Disponível em: http:// www.nhssurveys.org/surveys/660

34. Esperidião M, Bomfim LA. Avaliação de satisfação de usuários. Cien Saude Colet 2005; 10(Supl.):303-312.

Artigo apresentado em 03/06/2016

Aprovado em 11/11/2016

Versão final apresentada em 13/11/2016 